\title{
Behavioral and In Vivo Electrophysiological Evidence for Presymptomatic Alteration of Prefrontostriatal Processing in the Transgenic Rat Model for Huntington Disease
}

\author{
Sophie Höhn, ${ }^{1,2 *}$ Glenn Dallérac, ${ }^{1,2 *}$ Alexis Faure, ${ }^{1,2 *}$ Yvonne K. Urbach,,${ }^{4}$ Huu Phuc Nguyen, ${ }^{3}$ Olaf Riess, ${ }^{3}$ \\ Stephan von Hörsten, ${ }^{4}$ Pascale Le Blanc, ${ }^{1,2}$ Nathalie Desvignes, ${ }^{1,2}$ Nicole El Massioui, ${ }^{1,2}$ Bruce L. Brown, ${ }^{5}$ \\ and Valerie Doyère ${ }^{1,2}$ \\ ${ }^{1}$ Université Paris-Sud, Centre de Neurosciences Paris-Sud, Unité Mixte de Recherche 8195, F-91405 Orsay, France, ${ }^{2}$ Centre National de la Recherche \\ Scientifique, F-91405 Orsay, France, ${ }^{3}$ Department of Medical Genetics, University of Tübingen, D-72076 Tübingen, Germany, ${ }^{4}$ Experimental Therapy, Franz \\ Penzoldt Center, Friedrich Alexander University, D-91054 Erlangen-Nürnberg, Germany, and ${ }^{5}$ Graduate Center, City University of New York, and \\ Department of Psychology, Queens College, Flushing, New York 11367
}

Cognitive decline precedes motor symptoms in Huntington disease (HD). A transgenic rat model for HD carrying only 51 CAG repeats recapitulates the late-onset $\mathrm{HD}$ phenotype. Here, we assessed prefrontostriatal function in this model through both behavioral and electrophysiological assays. Behavioral examination consisted in a temporal bisection task within a supra-second range ( $2 v \mathrm{~s} .8 \mathrm{~s}$ ), which is thought to involve prefrontostriatal networks. In two independent experiments, the behavioral analysis revealed poorer temporal sensitivity as early as 4 months of age, well before detection of overt motor deficits. At a later symptomatic age, animals were impaired in their temporal discriminative behavior. In vivo recording of field potentials in the dorsomedial striatum evoked by stimulation of the prelimbic cortex were studied in 4- to 5-month-old rats. Input/output curves, paired-pulse function, and plasticity induced by theta-burst stimulation (TBS) were assessed. Results showed an altered plasticity, with higher paired-pulse facilitation, enhanced short-term depression, as well as stronger long-term potentiation after TBS in homozygous transgenic rats. Results from the heterozygous animals mostly fell between wild-type and homozygous transgenic rats. Our results suggest that normal plasticity in prefrontostriatal circuits may be necessary for reliable and precise timing behavior. Furthermore, the present study provides the first behavioral and electrophysiological evidence of a presymptomatic alteration of prefrontostriatal processing in an animal model for Huntington disease and suggests that supra-second timing may be the earliest cognitive dysfunction in HD.

\section{Introduction}

Huntington disease (HD) is a neurodegenerative disease attributable to the expansion of a CAG repeat within the first exon of the huntingtin gene. Classical symptoms have been described as a triad of motor, cognitive, and emotional disorders (Wilson et al., 1987). Interestingly, presymptomatic carriers have been reported to exhibit subtle cognitive alterations that appear mainly executive in nature (e.g., attentional set

\footnotetext{
Received March 10, 2011; accepted April 22, 2011.

Author contributions: S.H., G.D., A.F., Y.K.U., H.P.N., O.R., S.v.H., N.E.M., B.L.B., and V.D. designed research; S.H., G.D., A.F., Y.K.U., H.P.N., O.R., S.v.H., P.L.B., N.D., N.E.M., B.L.B., and V.D. performed research; S.H., G.D., A.F., Y.K.U., H.P.N., O.R., S.v.H., N.E.M., B.L.B., and V.D. analyzed data; S.H., G.D., A.F., Y.K.U., H.P.N., O.R., S.v.H., N.E.M., B.L.B., and V.D. wrote the paper.

*S.H., G.D., and A.F. contributed equally to this work.

This work was supported by European Union Sixth Framework Program Promemoria, RATstream Life Sciences and Health Grant LSH-037846, and Agence Nationale pour la Recherche Grant ANR-07-48 Memotime. S.H. was supported by the Ministry of Research. B.L.B. was supported by Professional Staff Congress-City University of New York Grant 61336-0039.

Correspondence should be addressed to Dr. Valérie Doyère, Centre de Neurosciences Paris-Sud, CNRS, UMR 8195, Université Paris-Sud, Bat. 446, 91405 Orsay, France. E-mail: valerie.doyere@u-psud.fr.

DOI:10.1523/JNEUROSCI.1238-11.2011

Copyright $\odot 2011$ the authors $\quad 0270-6474 / 11 / 318986-12 \$ 15.00 / 0$
}

shifting and decision-making deficiencies) (Foroud et al., 1995; Lawrence et al., 1996, 1998).

Progressive neurodegeneration of the GABAergic mediumspiny neurons of the striatum is a hallmark of the disease (Vonsattel et al., 1985). The striatum receives glutamatergic cortical inputs from the entire cerebral cortex in a topographic manner (Gerfen, 1984). Most neurophysiological studies of HD models have been performed in vitro, precluding the characterization of specific cortical afferents dysfunction. The few studies performed in vivo reported reduced plasticity in the motor cortex of symptomatic patients (Crupi et al., 2008) and dysregulated firing patterns of striatal cells in symptomatic rodents (Miller et al., 2008, 2010). Such impaired firing pattern was also detected in the prefrontal cortex of symptomatic R6/2 mice (Walker et al., 2008). Although it has been suggested that HD may be described as a frontostriatal dementia (Lawrence et al., 1996), whether early impairment in executive functions is linked to a deficiency in frontostriatal connectivity has not yet been addressed.

Prefrontostriatal circuits are thought to play a critical role in temporal processing (Buhusi and Meck, 2005). The rare HD studies examining timing have focused on durations under $1.2 \mathrm{~s}$ (Woodruff-Pak and Papka, 1996; Paulsen et al., 2004; Beste et al., 
2007; Hinton et al., 2007), a range that involves automatic, more than cognitive, processing (Rammsayer, 1999; Grondin, 2001). Notably, disrupted motor timing in presymptomatic patients correlates with the number of years from estimated onset of overt symptoms (Hinton et al., 2007). To our knowledge, the only study evaluating the timing of supra-second durations (20 s) used a peak-interval task in symptomatic HD mice and showed reduced temporal control with flatter peak functions (Balci et al., 2009). However, this time production procedure has inherent time-dependent motor components and is sensitive to deficient inhibitory control of behavior, therefore precluding assessment unique to temporal capabilities (Matell et al., 2006; Meck, 2006). Thus, in the present study, we sought to determine whether disruption of temporal processing in the supra-seconds range is one of the presymptomatic impeded cognitive functions and whether it could be associated with early frontostriatal alterations. We used a transgenic rat model of HD with 51 CAG repeats (tgHD) (von Hörsten et al., 2003) that closely resembles the human lateonset HD phenotype. Indeed, the first motor symptoms appear at $\sim 6$ months of age and striatal neurodegeneration from 8 months onward (von Hörsten et al., 2003; Nguyen et al., 2006). Here, using a 2 versus $8 \mathrm{~s}$ bisection task, a time perception procedure in which correct signal classification is not necessarily dependent on the timing of a choice response (Cheng et al., 2006, 2007), we show that $\operatorname{tgHD}$ rats have poorer temporal sensitivity as early as 4 months of age. In vivo recordings of field potentials (FPs) evoked in the dorsomedial striatum (DMS) by stimulation of the prefrontal cortex suggest that this disruption in executive function reflects modified plasticity of prefrontostriatal afferents.

\section{Materials and Methods}

Here we report four independent series of experiments. The first study consisted of assessing temporal discrimination in homozygous transgenic animals and their wild-type (wt) controls as a function of age (from 3 to 12 months of age) in a longitudinal design. Because the temporal task used a tone as the discriminative timing cue, the second study controlled for intact auditory processing in homozygous transgenic animals and wild-type controls during the first year of lifespan. The third study aimed at replicating the presymptomatic timing deficits and assessing the dependence on genotype by testing heterozygous transgenic animals in addition to homozygous and wild-type rats. The fourth study assessed presymptomatic function through in vivo electrophysiological recordings of prefrontostriatal field potentials.

\section{Temporal discrimination as a function of age \\ Animals}

This first series of experiments was performed on 21 male, 9 wt $(-/-)$, and $12 \operatorname{tgHD}(+/+)$ rats that were imported in our colony at 3 months of age and housed in pairs of same genotype in a temperature- and humidity-controlled colony room $\left(23^{\circ} \mathrm{C}, 41 \%\right.$ humidity). The transgenic $\mathrm{HD}$ rat carries a truncated huntingtin cDNA fragment with $51 \mathrm{CAG}$ repeats under the control of the rat huntingtin promoter (von Hörsten et al., 2003). The genetic background of the transgenic HD rat is derived from the Sprague Dawley strain (outbred). A $12 \mathrm{~h}$ light/dark cycle was maintained during the experiment (light on at 8:00 A.M.). On arrival in the laboratory, rats were given access to food and water ad libitum for 2 weeks and handled on a daily basis. Food was then progressively reduced until rats reached $80 \%$ of their initial weight. Throughout the experiment, rats were fed a daily ration just after the behavioral task, for them to be maintained at $85 \%$ of their normal free-feeding weight. All experiments were performed in accordance with the recommendations of the European Economic Community (86/609/EEC) and the French National Committee (87/848) for care and use of laboratory animals.

\section{Apparatus}

Soundproofed chambers (Coulbourn Instruments/Harvard Apparatus) were used for the temporal training task. Lever boxes $(50.8 \mathrm{~cm}$ long $X$
$63.5 \mathrm{~cm}$ wide $\times 66 \mathrm{~cm}$ high) were constructed with heavy-duty aluminum (floor and roof), and the walls were made of plastic and transparent Plexiglas. A pellet dispenser delivered food pellets ( $45 \mathrm{mg}$ grain-based precision pellets) to a food cup located on the left wall of the box. Two 4 $\mathrm{cm}$ retractable response levers (H23-17RA; Coulbourn Instruments/ Harvard Apparatus) were situated at each side of the food cup and horizontally placed $2.5 \mathrm{~cm}$ above the grid floor. The chamber floor was composed of 15 stainless steel rods spaced $1.7 \mathrm{~cm}$ apart. A speaker was located on the side of the box and permitted delivery of an auditory stimulus $(1 \mathrm{kHz}, 80 \mathrm{~dB})$. Each lever box was housed in a ventilated chamber, with a $65 \mathrm{~dB}$ background noise. At the beginning of each session, a red light was turned on. Chambers were controlled by means of a computer that recorded behavioral responses with the Graphic State software (Coulbourn Instruments/Harvard Apparatus) and a temporal precision of $20 \mathrm{~ms}$.

\section{Behavioral procedures}

The longitudinal study was conducted from 3 to 12 months. It was composed of a temporal discrimination training phase, followed by bisection tests repeated each month. At 9 months, rats were submitted to a modified staircase task, followed by a reversal task between 10 and 11 months, and the experiment ended with bisection tests at 12 months of age.

Animals were run at the same time each day in six cohorts with approximately the same number of transgenic and wt rats. Each rat was assigned to a given lever box from the beginning to the end of the experiment.

Pretraining. The pretraining phase started at 3 months of age with a magazine training session in which 30 pellets were delivered with a mean intertrial interval (ITI) of $60 \mathrm{~s}$ (variable intervals ranging between 20 and $100 \mathrm{~s}$ ). On the second day, rats had one session of continuous reinforcement with the left lever, and next day one session with the right lever. The session ended when rats obtained 50 reinforcers. If this criterion was not met within $30 \mathrm{~min}$, an extra session was run at the end of the day.

Temporal discrimination training. The 2 versus $8 \mathrm{~s}$ temporal discrimination training was implemented as described previously (Callu et al., 2009). It consisted of $3 \mathrm{~d}$ of $100 \%$ forced-choice trials, $3 \mathrm{~d}$ of $50 \%$ freechoice trials, followed by $11 \mathrm{~d}$ of $100 \%$ free-choice trials. The forcedchoice trials consisted of the presentation of only the correct lever (left or right) associated with the corresponding tone duration ( 2 or $8 \mathrm{~s}$ ), whereas both levers were presented on free-choice trials. The tone stimulus was presented for each duration with equal probability in random order in two blocks of 40 trials, for a total of 80 trials. A constraint on the random order was that no more than three trials of a given duration could occur successively. On each session, the same random order was used for all the animals. For half of the animals in each group (transgenic and wild type), a response on the left lever was reinforced after the short duration, and a response on the right lever was reinforced after the long duration; for the remaining rats, the relation of tone duration and reinforced response location was reversed. The ITI ranged from 20 to $40 \mathrm{~s}$, with a mean of $30 \mathrm{~s}$. The lever was retracted immediately after a response. In the absence of a response, the lever was retracted $10 \mathrm{~s}$ after tone-stimulus termination for the first $2 \mathrm{~d}$ of $100 \%$ forced-choice training and $5 \mathrm{~s}$ thereafter.

Bisection 1 tests. After discrimination training, a psychophysical choice procedure was conducted with five intermediate durations $(2.5,3.2,4,5$, and $6.3 \mathrm{~s}$ ) without reinforcement (12 trials each), in addition to the two training durations ( 2 and $8 \mathrm{~s}, 60$ trials of each) with reinforcement. The order of trials was randomized within two blocks of 90 trials (60 training trials and six test trials for each of the five durations). The ITI varied between 20 and $40 \mathrm{~s}$, with a mean of $30 \mathrm{~s}$. The bisection task was conducted $4-5 \mathrm{~d}$ each month, from 4 to 8 months, and was always followed by $1 \mathrm{~d}$ of discrimination training. The longitudinal design was intended to follow the changes in temporal performance as the disease progresses in the transgenic animals. However, across sessions, tgHD and wt rats stopped pressing levers for the intermediate nonreinforced durations of the bisection task, which precluded the analysis of psychometric functions (our unpublished observations).

Reduction of anchor duration difference. At 9 months, to estimate an optimal anchor duration difference that would prevent the rats from failing to respond to intermediate signal duration in later bisection tests, 
we exposed the animals to a progressive reduction of the difference between the short and long cue durations in approximately equal log steps across sessions $(2.41-6.65,2.64-6.06,2.89-5.53,3.17-5.04,3.48-4.59$, and $3.82-4.19 \mathrm{~s})$ in the temporal discrimination task. We conducted 12 sessions, with two daily sessions per step ( 40 trials of each, short and long, duration per session). All trials with a correct response were reinforced.

Reversal. At 10 months, we retrained the rats in the temporal discrimination task with 2 and $8 \mathrm{~s}$ tone durations ( 12 sessions, two sessions per day) and then reversed the duration/lever correspondence. The reversal training was conducted for $8 \mathrm{~d}$, with two sessions per day. As in the initial choice procedure task, 40 trials were associated with the short duration and 40 trials with the long duration.

Bisection 2. At 12 months, rats were retrained in the temporal discrimination task ( 10 sessions in $5 \mathrm{~d}$ ) and tested with the bisection procedure (four sessions) with a smaller range of durations chosen from the data obtained previously when anchor duration differences were reduced. The two training durations were 2.64 and $6.06 \mathrm{~s}$, and the five intermediate durations were $3.03,3.48,4,4.59$, and $5.28 \mathrm{~s}$.

\section{Data analysis}

Response location and latency were recorded for each trial. Discrimination data were analyzed as percentage of correct responses across all trials. Bisection test data were calculated as proportion of responses on the lever assigned as correct for the long-duration stimulus on all trials with response.

The function relating proportion of "long" responses to stimulus duration (bisection function) is typically sigmoidal in shape, an example of the well-known psychometric function. The stimulus value corresponding to $p_{\text {(long) }}=0.5$ is termed the point of subjective equality (PSE) and represents the stimulation duration that has been interpreted as subjectively equidistant from 2 and $8 \mathrm{~s}$ training values (bisection point). Previous research in rats (Church and Deluty, 1977; Maricq et al., 1981; Maricq and Church, 1983) has found the PSE to be positioned at the geometric mean of the two extreme durations, for which correct responses were reinforced (i.e., $4 \mathrm{~s}$, for $2 \mathrm{~s}$ and $8 \mathrm{~s}$ training values). The slope of the function in the vicinity of the PSE reflects temporal sensitivity. The bisection functions were averaged across sessions within each age for each rat and analyzed with the pseudologistic model fit (Killeen et al., 1997) using Prism software (GraphPad Software) and assuming negligible contribution of constant and Poisson sources of variability (Allan, 2002). The proportion of variance accounted for by the fit, the PSE, and the temporal sensitivity parameter (gamma) were estimated for each rat. Gamma, which is proportional to the Weber fraction, is inversely related to temporal sensitivity.

\section{Prepulse inhibition test as a function of age}

We tested whether any potential disruption in timing behavior could possibly be explained by differences in processing auditory stimuli by using prepulse inhibition (PPI) of the startle reflex (SR). The PPI procedure was aimed at testing the level of reduction of the magnitude of the acoustic startle by previous presentation of the prepulse stimulus. In the present experiment, 22 animals $(11-/-$ and $11+/+)$ were tested in cohorts of four in the startle response system in a longitudinal design from 1 to 12 months of age.

\section{Apparatus and paradigm}

SR was measured with four TSE Startle Response Systems (TSE Systems), as described previously (Karl et al., 2003; Urbach et al., 2010). Wire-mesh cages $(27 \times 9 \times 10 \mathrm{~cm})$ were fixed on a piezo-accelerometer that can determine weight alterations within milliseconds, in a sound-attenuated chamber equipped with two loudspeakers that delivered a constant white background noise of $68 \mathrm{~dB}$ sound pressure level. Habituation and baseline determination of activity for a duration of $2 \mathrm{~min}$ were followed by 10 startle trials with a defined pulse $(20 \mathrm{~ms}, 120 \mathrm{~dB})$, which were presented with ITIs of 6-12 s in random order (baseline determination component). For PPI assessment, animals received three different trial types in a random order: (1) 10 prepulses of $80 \mathrm{~dB}$ (duration, $20 \mathrm{~ms}$ ), followed after $100 \mathrm{~ms}$ by a startle pulse of $120 \mathrm{~dB}$ for $20 \mathrm{~ms}$ (PPI component); (2) three prepulses $(20 \mathrm{~ms}, 80 \mathrm{~dB}$ ) without startle pulse (prepulse-alone component); (3) 15 pulses of $120 \mathrm{~dB}$ (20 ms) alone (SR component). All trials were programmed with an intertrial interval randomly set at 6 or $12 \mathrm{~s}$. The whole session lasted $\sim 14 \mathrm{~min}$.

\section{Data analysis}

SR response intensities were averaged and expressed as arbitrary units. PPI was calculated as a percentage score: $\left(100 \times\left(\right.\right.$ PPI $\left.\left.{ }^{\star} 100\right) / S R\right)$.

\section{Temporal sensitivity at presymptomatic age as a function of genotype}

Animals

In this second study, male transgenic homozygous $(+/+, n=8)$, heterozygous $(+/-, n=8)$, and wild-type $(-/-, n=8)$ rats obtained from an in-house tgHD colony (initially generated with 10 pairs of heterozygous rats) and genotyped in Germany as described previously (Kántor et al., 2006) were used. At the beginning of the study, rats were 4 months old and $\sim 400 \mathrm{~g}$ in weight. Animal care procedures, food deprivation, and experimental procedures were as described above unless stated otherwise.

\section{Apparatus}

Six lever boxes (Coulbourn Instruments/Harvard Apparatus) in soundproofed chambers were used. The orientation of the equipment was completely reversed (e.g., levers on the right wall) for two of the chambers. Animal-box assignment was counterbalanced between groups.

\section{Behavioral procedure}

In this study, rats were first submitted to a 2 versus $8 \mathrm{~s}$ temporal discrimination task, equivalent to the discrimination protocol described above, at 4.5 months of age. To determine the anchor duration for a personalized version of the bisection protocol, a staircase procedure was implemented at 5.5 months of age, and testing on the personalized bisection procedure was conducted at 6.5 months. An emotion study with the bisection task (not reported here) was subsequently conducted, and finally, at $\sim 7$ months of age, rats were challenged with a progressive reduction across sessions in the difference between anchor durations in the bisection task.

Temporal discrimination training. Temporal discrimination training consisted of $2 \mathrm{~d}$ of $100 \%$ forced-choice trials, followed by $50 \%$ freechoice sessions until the criterion of $1 \mathrm{~d}$ with $80 \%$ correct responses in the free-choice trials was reached for each rat. Next, a 100\% free-choice procedure was run until a criterion of $2 \mathrm{~d}$ with $85 \%$ correct responses was attained. Free-choice sessions consisted of 120 trials (60 trials at each duration).

Staircase procedure. At 5.5 months of age, animals were submitted to a modified staircase procedure. In that protocol, 15 possible short-long anchor pairs were available in the same session with progressive reduction in the difference between short and long cue durations in logarithmic steps (starting at 2-8 s and ending at 3.91-4.09 s). All correct responses were reinforced. Sessions comprised 180 trials in blocks of a maximum 12 trials, with the rule for an increase, decrease, or no change in step set at 75\% correct responses. Each day, each rat started the session with a discrimination set at a difficulty one step easier than that at the end of the session on the previous day. On each session, we calculated a mean stimulus duration difference based on step values from all the trials. The criterion for staircase stability was that this value did not change between sessions $>5 \%$ in three sessions, with one reversal of sign, with the mean defining the individual difference limen (DL). When criterion was reached, one additional final session was run. Animals were run for a maximum of 26 sessions.

Personalized bisection. Analysis of the last $4 \mathrm{~d}$ of staircase performance provided an estimate of DL for each rat, thereby enabling estimation of the individual values for anchor (short and long) tone durations that would support a level of $80 \%$ correct responses. With this personalization procedure, we equalized the difficulty of discrimination across animals and genotypes. Using these personalized anchor values, the rats were retrained in the temporal discrimination task for $2-3 \mathrm{~d}$ to verify that their performance met $\sim 80 \%$ of correct responses. Next, a bisection procedure was implemented with five intermediate durations (12 trials 
each), in addition to the two anchor durations (short and long, 60 trials of each), comprising 180 trials in total. The intermediate durations were calculated to be equally spaced between the personalized anchor durations along a logarithmic scale. The ITI varied between 10 and 80 s, with a mean of $30 \mathrm{~s}$.

Anchor difference reduction. Rats were first retested in a single session of personalized bisection as described above. Then, the difference between anchor durations was progressively reduced in two-session blocks, corresponding to six levels of estimated difficulty, i.e., 75, 70, 65, 60, and $55 \%$ of correct responses, with respect to the DL calculated during the staircase procedure (see above).

\section{Data analysis}

Discrimination and bisection data were analyzed as in the previous set of behavioral experiments (see above).

\section{Neurophysiological assessment of presymptomatic prefrontostriatal functioning \\ In vivo electrophysiological characterization of the prefrontodorsostriatal pathway}

The prelimbic area (PL) of the prefrontal cortex is known to project to the dorsal striatum, in majority to its medial part (Gabbott et al., 2005). However, this pathway has never been characterized electrophysiologically in the rat in vivo. Therefore, we first performed an initial electrophysiological characterization of the field potentials evoked in the DMS by stimulation of the PL of the prefrontal cortex on normal young adult male Sprague Dawley rats (300-400 g; Charles River).

Surgery. The animals were prepared for the operation under sodium pentobarbitone anesthesia $(0.1 \mathrm{ml} / 100 \mathrm{~g}$, i.p., supplemented with $0.2 \mathrm{ml}$ of a dilution of $50 \%$ if necessary; Ceva Santé Animale), a dilution of $10 \%$ of Tolfedine $(0.1 \mathrm{ml} / 100 \mathrm{~g}$, s.c.; Vétoquinol), and $0.2 \mathrm{ml}$ of atropine (intraperitoneally; Aguettant). The body temperature was maintained during the experiment at $37^{\circ} \mathrm{C}$ with an electric blanket to avoid hypothermia. Conventional surgical techniques were used, and coordinates were chosen according to the atlas of Paxinos and Watson (1986).

One recording electrode (nichrome, $65 \mu \mathrm{m}$ in diameter) attached to a guide tube and extending from it by $\sim 0.5 \mathrm{~mm}$ was placed in the dorsomedial striatum [anteroposterior (AP), $1.2 \mathrm{~mm}$; lateral (L), $-2 \mathrm{~mm}$ ]. One bipolar concentric stimulating electrode ( $250 \mu \mathrm{m}$ in diameter) was positioned in the prelimbic area of the prefrontal cortex (AP, $3.2 \mathrm{~mm}$; L, $-0.3 \mathrm{~mm}$ ). A cortical silver ball, placed contralaterally on top of the visual cortex (AP, $-7 \mathrm{~mm}$; $\mathrm{L}, 2 \mathrm{~mm}$ ), served as ground and reference. Histological verification of recording and stimulating electrode placements was performed on some rats. At the end of the experiment, rats were anesthetized with an overdose of sodium pentobarbitone $(1 \mathrm{ml})$ and perfused transcardially with $4 \%$ paraformaldehyde. Coronal sections were cut at $40 \mu \mathrm{m}$ on a microtome and mounted onto glass slides. Sections were then examined using an Olympus BX60 fluorescent-imaging microscope (Leica).

PL-DMS field potential characteristics. Characteristics of the PL-DMS field potential are summarized in Figure 1. Maximal amplitude of the negative field potentials was obtained by stimulation of the dorsal part of the prelimbic cortex and recording in the dorsomedial part of the striatum (Fig. 1 $A, B$ ). Optimal depths were found to be at $\sim 2.5$ and $3.5 \mathrm{~mm}$ below dura for stimulating and recording electrodes, respectively. The mean slope value was $0.17 \pm 0.02 \mathrm{~V} / \mathrm{s}(n=10)$, and the mean peak latency was $13.37 \pm 0.48 \mathrm{~ms}$.

The field response was composed of both glutamatergic and GABAergic components (Fig. 1C) with spike discharges at the time of the peak of the response. Blockade of AMPA receptors with local infusion of DNQX (1 mM, $0.5 \mu \mathrm{l}$ ) indeed produced the progressive disappearance of the negative response and spikes and the appearance, at the same latency of a positive response (Fig. $1 C$, middle). Furthermore, addition of the GABA receptor antagonist picrotoxin $(1 \mathrm{mM}, 0.5 \mu \mathrm{l})$ resulted in the disappearance of the latter positive component (Fig. $1 C$, right). Thus, the extracellular field potential most likely represents a mixture of depolarization (EPSP) and hyperpolarization (IPSP), which can be analyzed through the initial slope of the response, and spikes mainly reflected in the response amplitude.
The field potentials routinely followed a train of high-frequency stimulation $(50 \mathrm{~Hz})($ Fig. $1 \mathrm{E})$, indicating that there were monosynaptic and not the result of polysynaptic activation. This was confirmed by recording of an antidromic response in the prelimbic cortex evoked by stimulation of the mediodorsal striatum (Fig. 1D).

\section{Electrophysiological assessment of prefrontostriatal synaptic}

function in transgenic animals

Animals and surgery. This series of experiments was conducted in vivo under anesthesia on $12 \mathrm{wt}(-/-), 19$ homozygous $(+/+)$, and 9 heterozygous $(+/-)$ transgenic rats aged 3.5 to 5.5 months old, weighing from 300 to $500 \mathrm{~g}$. They were prepared for surgery and electrophysiological recordings as described above. Final depths of the electrodes were determined using electrophysiological guidance to maximize the evoked response and were $\sim 4 \mathrm{~mm}$ below the dura for the recording electrode (mean of 4.2, 4.1, and $4.1 \mathrm{~mm}$ for wt, heterozygous, and homozygous, respectively) and $2.5 \mathrm{~mm}$ below the dura for the stimulation electrode (mean of 2.6, 2.5, and $2.5 \mathrm{~mm}$ for wt, heterozygous, and homozygous, respectively).

Electrophysiological recording procedure. FPs evoked by stimulation of the prelimbic cortex (monophasic square pulse, $80 \mu \mathrm{s}$ ) were recorded. Signals were amplified $(1000 \times$ ), filtered (bandpass, $0.1-3 \mathrm{kHz}$ ), digitized at $10 \mathrm{kHz}$, and stored on disk for offline analyses. Stimulation of the pathway and recording was automated using the program Advance (Copyright 1988-1997, Robert McKellar Douglas; McKellar Designs and University of British Columbia).

After stabilization of the preparation, input/output curves (I/O) were generated by collecting responses to a series of 12 increasing current intensities $(80-800 \mu \mathrm{A})$. FPs were evoked at $0.07 \mathrm{~Hz}$, for a total of four times at each intensity. The test intensity used in all subsequent recordings was set to evoke FPs of $\sim 50 \%$ of its maximum amplitude, and the intensity was chosen at $80 \%$ for the tetanic stimulation phase. Short-term plasticity was examined using a paired-pulse stimulation (PP) protocol that consisted of two stimulations with seven increasing intervals (20$1000 \mathrm{~ms}$ ) for a total of four times at each interval. Short- and long-term plasticity was subsequently assessed. After a baseline recording of $30 \mathrm{~min}$ (60 FPs, $0.03 \mathrm{~Hz}$ ), a theta-burst stimulation (TBS) consisting in three series of 10 trains $(100 \mathrm{~Hz}, 100 \mathrm{~ms})$ at $5 \mathrm{~Hz}$, with a 10 s interseries interval, was administered. Both 5 and $100 \mathrm{~Hz}$ stimulation have been used to induce striatal plasticity recorded intracellularly (Charpier and Deniau, 1997; Charpier et al., 1999). We chose to use a theta-burst stimulation paradigm, which combines both frequencies, to optimize our chance of inducing long-term plasticity in the striatum. Test recordings were resumed after TBS for a 60 min period, at the end of which PP and I/O profiles were again completed.

\section{Data analysis}

The initial slope and the amplitude were measured for each FP. Values were averaged across four tests for descriptive purposes and statistical comparisons. Averaged values were normalized for each animal with respect to the mean value obtained during the baseline before the TBS. Paired-pulse ratios were calculated by dividing the averaged value obtained for the second pulse by the averaged value obtained for the first reference pulse (P2/P1). Paired-pulse facilitation is thus indicated by a ratio above 1 .

Statistical analyses for all data were performed with Student's $t$ tests or contrast ANOVAs (Rouanet et al., 1990), based on the a priori hypothesis that homozygous animals may differ from wt controls and that heterozygous animals may show intermediate results. An $\alpha$ level of 0.05 was used as the criterion for statistical significance.

\section{Results}

\section{Temporal discrimination as a function of age}

Performances during initial temporal discrimination training were analyzed with two dependent measures: percentage of correct responses during the free-choice trials and response latencies (Fig. 2A). Both measures showed discrimination learning with an increase in percentage correct responses and a decrease in response latencies across sessions $\left(F_{(10,190)}=7.39, p<0.001\right.$ and 


\section{A}

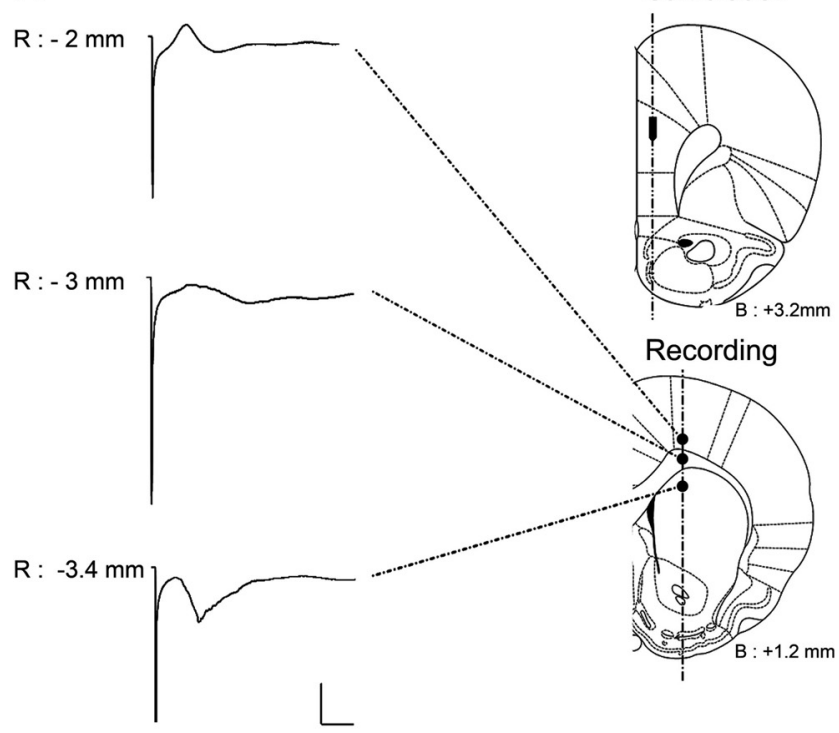

C

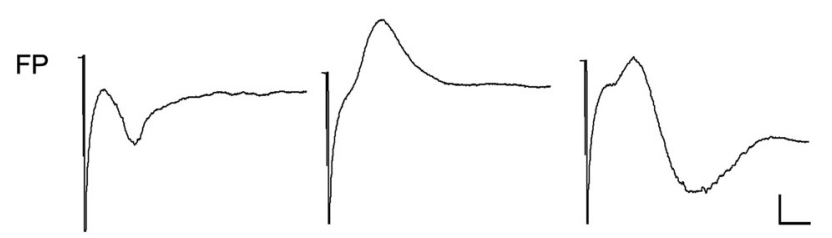

MUA IN
B

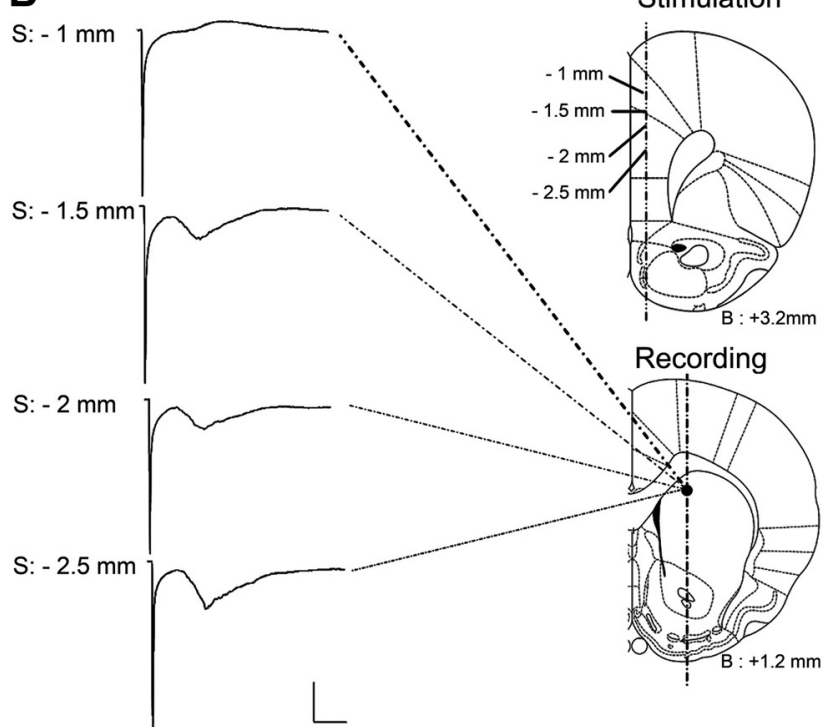

D

Orthodromic

Antidromic
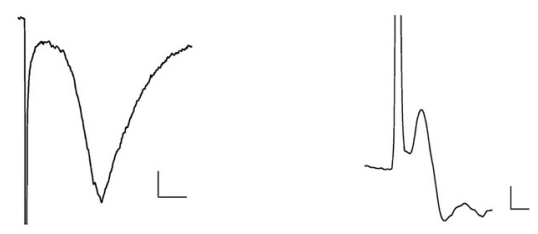

E

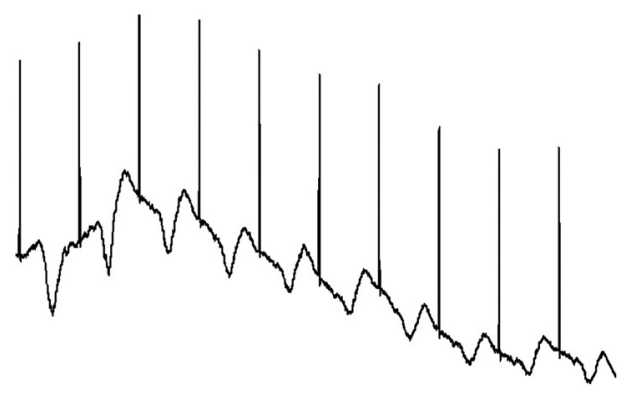

Figure 1. Characteristics of the field potentials evoked in the dorsal striatum by prefrontal cortex stimulations in vivo. $A$, Field potentials recorded during the lowering of the recording electrode at 2,3 , and $3.4 \mathrm{~mm}$ of depth below the dura, whereas the stimulation electrode is placed at the level of the dorsal part of prelimbic cortex. Calibration: $0.5 \mathrm{mV}, 5 \mathrm{~ms}$. $\boldsymbol{B}$, Field potentials recorded in the dorsal striatum during the lowering of the stimulation electrode at 1, 1.5, 2, and $2.5 \mathrm{~mm}$ of depth below the dura. Calibration: $0.5 \mathrm{mV}, 5 \mathrm{~ms}$. C, Glutamatergic and GABAergic components in the evoked field potentials; examples of simultaneous recording of FPs (calibration: $0.25 \mathrm{mV}, 5 \mathrm{~ms}$ ) and multiunit activity (MUA; calibration: $2.5 \mathrm{mV}, 5 \mathrm{~ms}$ ) evoked by test stimulations. From Left to Right, Baseline conditions, after local infusion of DNQX $(1 \mathrm{~mm}, 0.5 \mu \mathrm{l})$, and after local infusion of DNQX + picrotoxin (PTX; $1 \mathrm{~mm}, 0.5 \mu \mathrm{l})$. Multiunit was recorded in parallel to field responses to confirm that negative-going response indeed corresponds to an excitatory activity and embed a population spike as well as an fEPSP. $D$, Examples of orthodromic response recorded in the dorsomedial striatum (calibration: $0.05 \mathrm{mV}, 5 \mathrm{~ms}$ ) and antidromic response recorded in the prelimbic cortex after stimulation of the dorsomedial striatum (calibration: $0.05 \mathrm{mV}, 1 \mathrm{~ms}$ ). $\boldsymbol{E}$, Example of recording during 50 Hz stimulation (calibration: $0.2 \mathrm{mV}, 10 \mathrm{~ms}$ ).

$F_{(10,190)}=7.67, p<0.001$, respectively). Although a trend for a slight delay in learning the task was observed in the transgenic animals compared with the wt animals, no differences reached statistical significance, for either percentage correct responses (group difference, $F_{(1,19)}=2.07$, NS; group $\times$ session interaction, $F_{(10,190)}=1.20$, NS) or response latency (group difference, $F_{(1,19)}=1.01$, NS; group $\times$ session interaction, $\left.F_{(10,190)}=1.83, \mathrm{NS}\right)$.

At 4 months of age, both transgenic and wt rats showed normal bisection curves with proportion of responses on the lever assigned as correct for the long-duration stimulus increasing with the signal duration (Fig. $2 B$ ). As reported previously in similar conditions (Callu et al., 2009), response latencies decreased with increasing signal durations $\left(F_{(6,114)}=5.52, p<0.001\right)$ (Fig. $2 C$ ). The group effect and group $X$ duration interaction were not significant $\left(F_{(1,19)}=1.42\right.$, NS; and $F_{(6,114)}=1.32$, NS, respectively), showing that transgenic and wt animals were performing similarly. When psychometric functions were fitted, the proportion of variance accounted for by the fit was obtained for each rat. The $R^{2}$ values varied from 0.968 to 0.998 and from 0.971 to 0.998 for wt and tgHD groups, respectively. Two parameters (PSE and gamma) were estimated from the psychometric function of each individual, allowing quantitative estimation of the bisection point and temporal sensitivity, respectively. There was no difference in the PSE between tgHD and wt rats (Fig. 2D) (unpaired $t$ 
A

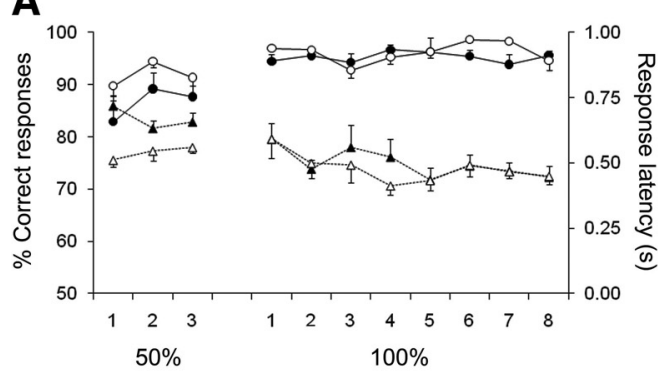

B
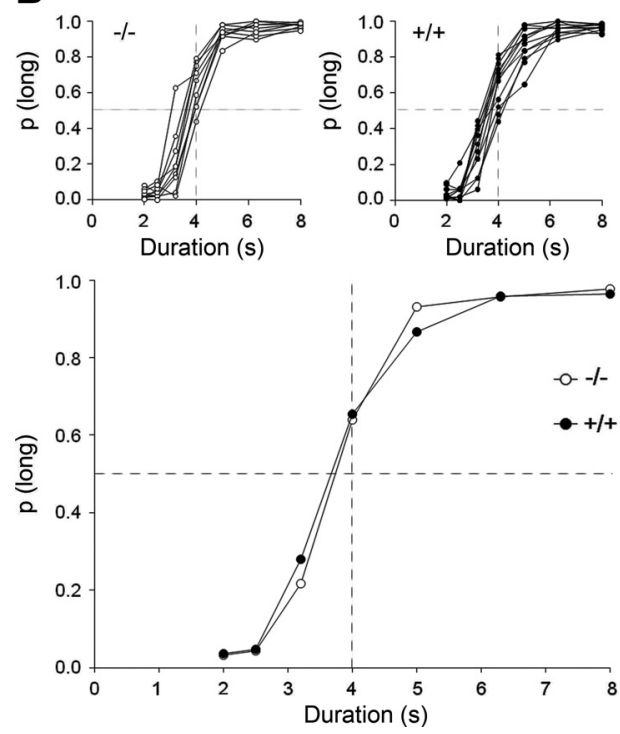

C

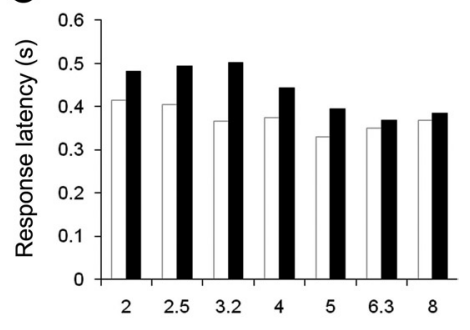

D

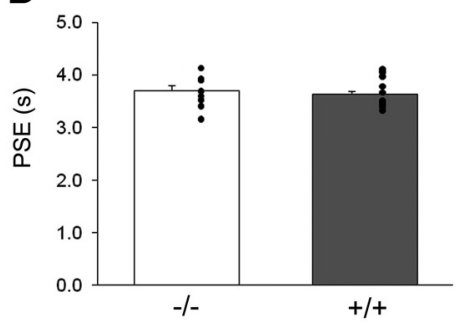

E

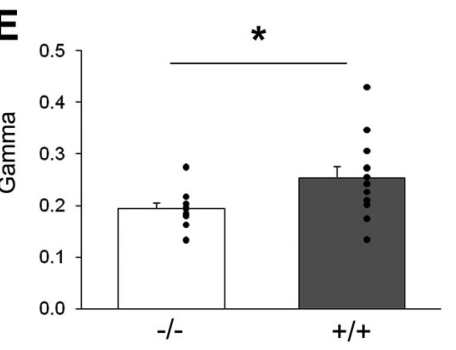

Figure 2. Temporal performance at 3-4 months of age for the transgenic HD rats $(+/+$, black) and their wt controls $(-/-$, white). $\boldsymbol{A}$, Acquisition of 2 versus 8 s temporal discrimination in $\operatorname{tg} \mathrm{HD}(n=12$, filled symbols) compared with their wt controls ( $n=$ 9 , open symbols) for both percentage correct responses (solid lines) and responses latencies (dotted lines). Performances during the training phase are depicted across sessions (the 3 first sessions are 50\% free-choice trials and the next 8 sessions are 100\% free-choice trials). $\boldsymbol{B}$, Bisection curves depict mean proportion of long responses as a function of signal duration for individual animals (insets) or group performances averaged across the five testing sessions conducted immediately after the last training session in temporal discrimination. $\boldsymbol{C}$, Mean response latencies as a function of signal duration during the bisection test. Note the decrease in latency as the signal duration increases. $\boldsymbol{D}, \boldsymbol{E}$, Mean (bars) and individual (dots) values for the extracted parameters of the fitted bisection function: PSE (D) and gamma $(\boldsymbol{E}) .{ }^{*} p<0.05$.
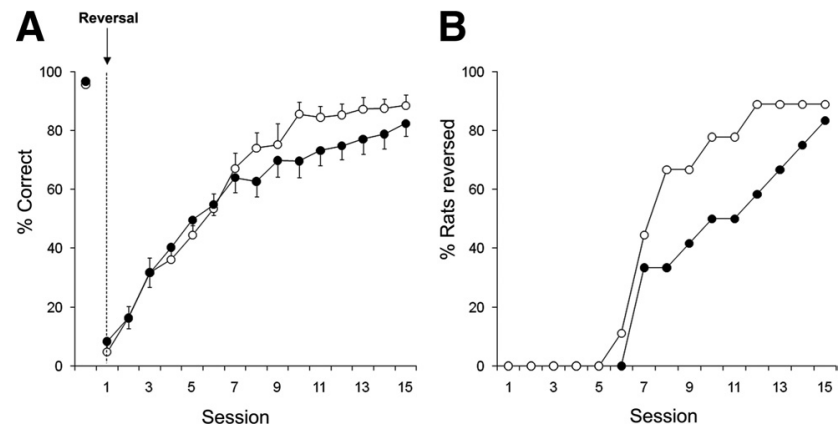

Figure 3. Mean \pm SEM percentage of correct responses $(\boldsymbol{A})$ and percentage of rats reversed $(\boldsymbol{B})$ across sessions at 10 months of age during reversal of the temporal discrimination task for the transgenic $\mathrm{HD}$ rats $(+/+$, filled circles) and their wt controls $(-/-$, open circles).

test for equal variances, $\left.t_{(19)}=0.439\right)$. In contrast, there was a striking difference between $\operatorname{tgHD}$ and wt rats in their temporal sensitivity (Fig. 2E). A significant increase in variance was observed in $\operatorname{tgHD}$ rats compared with wt ( $F$ test for equality of variance, $\left.F_{(8,11)}=4.06, p<0.03\right)$; taking the variance difference into consideration, the tgHD rats showed poorer temporal sen- sitivity evidenced through an increased mean gamma (unpaired $t$ test for unequal variances, $t_{(17)}=2.27, p<0.04$ ), which is indeed suggested by a shallower slope in the bisection curves (Fig. $2 \mathrm{~B}$ ).

At 10 months of age, a reversal of the 2 versus $8 \mathrm{~s}$ temporal discrimination task was achieved by interchanging the anchor durations and their corresponding levers. The effect of the reversal was observable from the first day (Fig. 3). Both groups of rats exhibited acquisition in the new task ( significant session effect, $F_{(14,266)}=$ $123.75, p<0.001)$. At the beginning of the reversal training, performances of both groups were equally disrupted (virtually $0 \%$ correct responses). Subsequently, however, tgHD rats were delayed compared with the wt rats in reaching the same terminal level of correct responses (Fig. $3 A$ ), and the percentage of rats scoring above $50 \%$ correct responses increased more slowly in the tgHD group compared with the wt group (Fig. $3 B$ ). On the percentage correct measure, difficulty in reversing for tgHD rats was confirmed by a significant group $\times$ session interaction $\left(F_{(14,266)}=2.10, p<0.02\right)$. These data therefore indicate that acquisition of the new rule was altered in the transgenic animals.

At 12 months of age, rats were tested on the bisection task with a reduced difference in anchor values to reduce duration discriminability and maintain responding at all intermediate durations (bisection 2). Response latencies did not differ significantly between groups (group, $F_{(1,19)}=2.99$, NS; group $\times$ duration interaction, $F<1$ ), reflecting a lack of gross motor deficit. Transgenic rats presented more variability in their individual bisection curves than did wt rats (Fig. 4A). Fitting the curves of the transgenic rats with the pseudologistic function resulted in a bimodal distribution for the proportion of variance accounted for $\left(R^{2}\right)$ (Fig. $4 B$ ). Considering only rats for which $R^{2}$ was higher than 0.8 , the averaged bisection curve for the tgHD group was shifted to the right compared with wt rats (Fig. 4C, left). There was a significant difference between $\operatorname{tgHD}$ and wt rats in mean PSE (Fig. $4 C$, middle) (unpaired $t$ test for equal variances, $t_{(16)}=2.84, p<0.02$ ). No difference in temporal sensitivity (gamma) between groups (Fig. $4 C$, right) (unpaired $t$ test for equal variances, $t_{(16)}=1.01$ ) was detected. Shifts in PSE indicate a mismatch between accumulator/working memory (online subjective duration) and reference memory. They have classically been interpreted as a change in either clock speed or memory alteration (Meck, 1983). A difference in clock speed is presumed to affect temporal behavior temporarily, because new reference values are continuously stored with repeated training. In contrast, a modification of temporal behavior attributable to memory alteration would emerge progressively with repetition. In the present experiment, it is unlikely that a difference in clock speed would have affected the PSE, because animals had been trained with these durations immedi- 
ately before the test, permitting adjustment of the reference memory to the prevailing contents of the accumulator/ working memory. Thus, the increase in PSE may rather reflect altered temporal memory.

\section{Auditory processing as a function of age}

Poorer temporal sensitivity for tgHD animals with auditory signals may conceivably result from a disruption in processing auditory stimuli rather than a deficit in timing per se. To verify that tgHD animals displayed intact auditory processing, we examined an independent group of tgHD rats in a PPI test of the startle reflex. This test is commonly used to assess impairments in auditory stimulus processing (for review, see Fitch et al., 2008).

Startle responses and prepulse inhibition as a function of age (from 1 to 12 months) are depicted in Figure 5. Startle amplitude in pulse-alone trials (Fig. 5A) increased significantly with age $\left(F_{(11,231)}=\right.$ 32.76, $p<0.0001)$, to reach a plateau from 6 months of age for both groups. There was no significant between-groups difference $\left(F_{(1,11)}=2.02\right.$, NS). tgHD and wt animals showed also increasing amplitude of PPI with age (Fig. $5 B)\left(F_{(11,231)}=\right.$ $4.58, p<0.0001)$, and no between-groups difference was observed $(F<1)$. These data show that short-duration auditory perception is normal in tgHD animals, indicating that the deficits observed in the bisection task are not attributable to differential auditory processing but reflect modifications in temporal behavior.

\section{Temporal sensitivity at presymptomatic age as a function of genotype}

As in the previous experiment, there was no difference in the speed of acquisition of the 2 versus $8 \mathrm{~s}$ temporal discrimination among groups. During temporal discrimination training, there was no genotype effect on the number of days to reach criteria in the $50 \%(F<1)$ or $100 \%\left(F_{(2,21)}=2.65\right.$, NS $)$ free-choice procedures.

In the staircase procedure, we restricted the analysis to the first $9 \mathrm{~d}$, before any animal met the stability criterion. As expected, a significant reduction in the mean stimulus duration difference $\left(F_{(8,168)}=32.00, p<0.001\right)$ was observed. There was no genotype effect either during the first $9 \mathrm{~d}\left(F_{(2,21)}=1.27\right.$, NS) or when considering only the last $4 \mathrm{~d}$ of staircase training used to calculate the personalized anchor durations in the bisection paradigm $\left(F_{(2,21)}=2.76\right.$, NS). The unexpected lack of difference between homozygous and wt DL may have been attributable to the fact that not all animals have reached the stability criterion and were not at an equivalent level of stabilized performances. In addition, the step sizes chosen were possibly not small enough to permit a sensitive assessment of the slight temporal disruption of temporal sensitivity (gamma) reported in the preceding experiment.
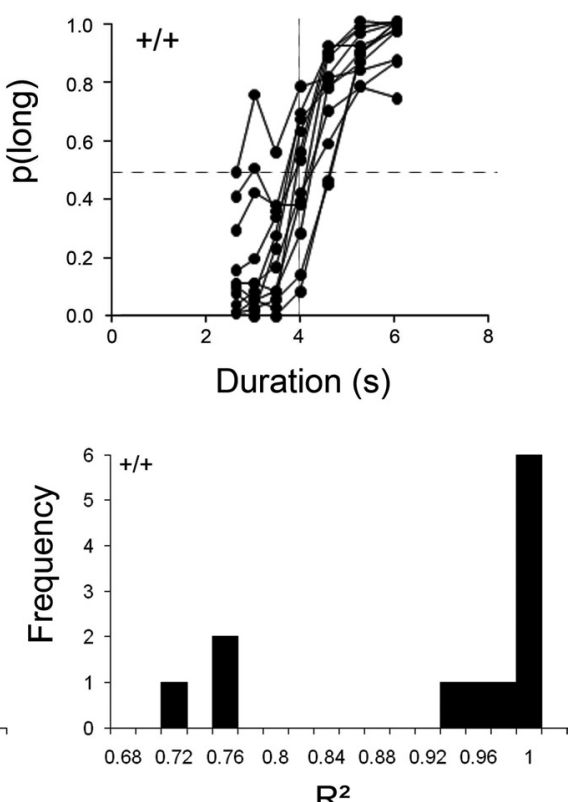

$\mathrm{R}^{2}$

$\mathrm{R}^{2}$
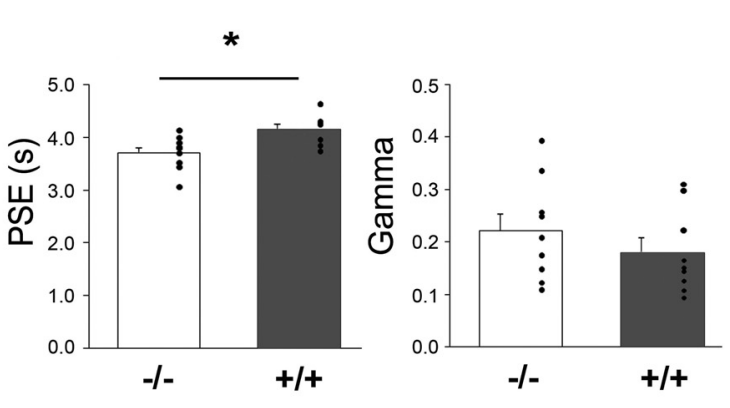

Figure 4. Performance during temporal bisection tests at 12 months of age (bisection 2$)$ for the transgenic $H D$ rats $(+/+$, ions for wt and tgHD rats. Middle and Right, Mean (bars) and individual (dots) values for the extracted parameters of the fitted bisection function, respectively: PSE and gamma. ${ }^{*} p<0.05$.
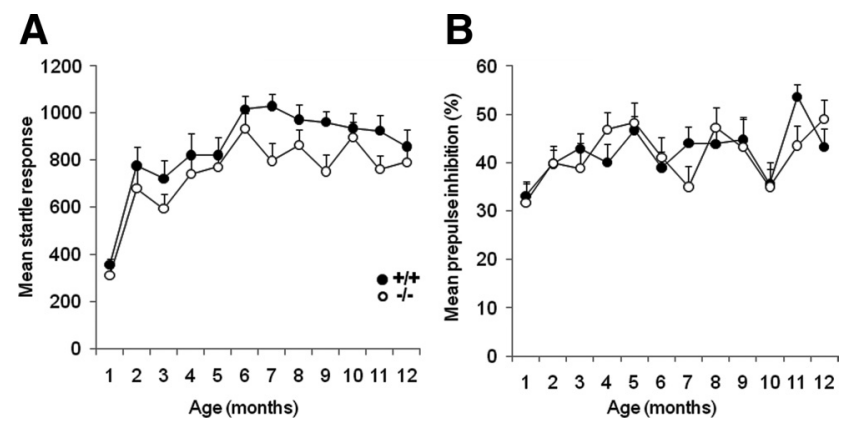

Figure 5. Auditory perception from 1 to 12 months of age for the transgenic HD rats $(+/+$, black) and their wt controls ( $-/-$, white). $A$, Startle response amplitude with a $120 \mathrm{~dB}$ pulse at different ages. $\boldsymbol{B}$, Inhibition of the startle reflex by a $80 \mathrm{~dB}$ prepulse.

The results obtained during the personalized bisection tests are shown in Figure 6. Because of a deviant bisection curve, a heterozygous outlier rat (based on Grubbs' test for both gamma and PSE values) was discarded from this analysis. In accordance with the data reported above in 4-month-old animals, homozygous 6-month-old rats showed a slightly shallower bisection curve compared with wt (Fig. 6A). Although a main effect of genotype was not obtained $\left(F_{(2,20)}=2.45, p<0.11\right)$, planned 
A

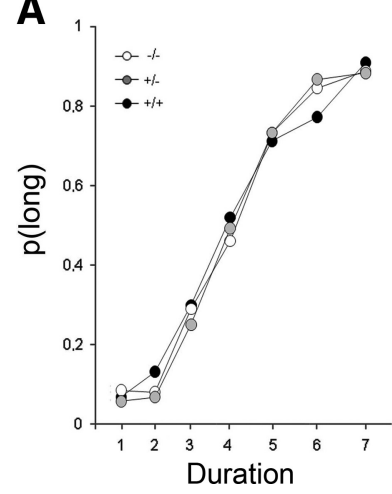

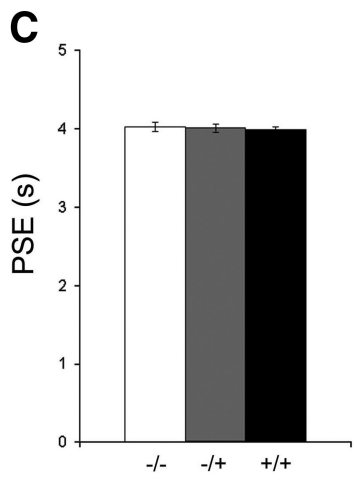

Figure 6. Performance during personalized bisection tests at 6 months of age for the homozygous $(+/+$, black), heterozygous $(+/-$, gray), and wt $(-/-$, white) rats. $\boldsymbol{A}$, Bisection curves depict mean proportion of long responses as a function of signal duration (1-7) averaged across the two sessions. Durations were chosen for each animal according to their performance during the staircase paradigm (see Materials and Methods). $\boldsymbol{B}, \boldsymbol{C}$, Mean \pm SEM values for the extracted parameters of the fitted bisection function: gamma (B) and PSE (C).

A

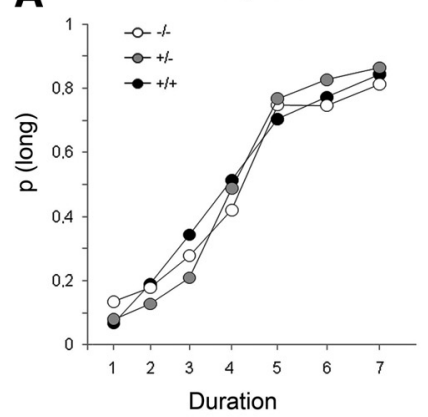

B

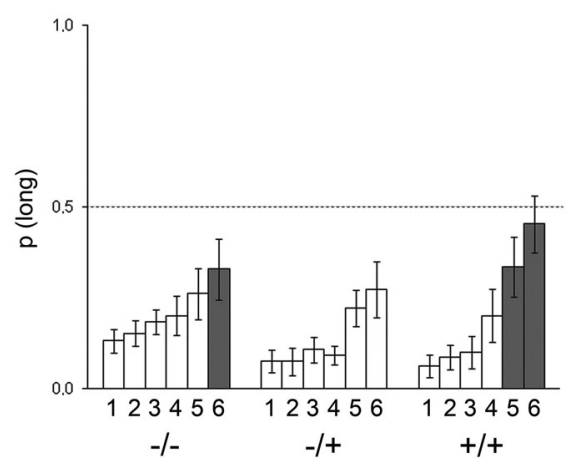

Difficulty level 6

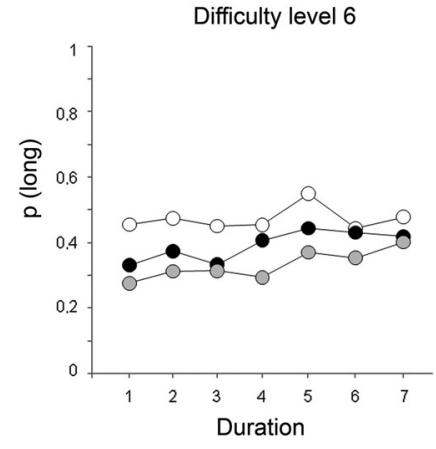

Long duration

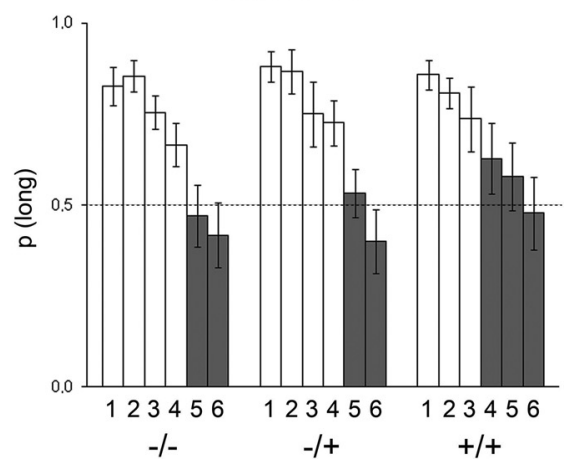

Figure 7. Performances when increasing the difficulty of the discrimination between signal durations during bisection tests. $\boldsymbol{A}$, Bisection curves representing the proportion of long responses for the easiest (left) and the hardest (right) levels of difficulty for the homozygous ( $+/+$, black), heterozygous ( +- , gray), and wt $(-/-$, white) rats. $\boldsymbol{B}$, Group mean \pm SEM proportion of long responses during the increasing six levels of difficulty for the short (left) and long (right) anchor durations. The gray bars highlight when performances are not statistically different from 0.5 , a value representing random-choice behavior.

(Fig. 7A), with the proportion of long responses increasing for the short-tone durations and decreasing for the long-tone durations. Notably, at both anchor durations, the homozygous tgHD reached the level of random-choice behavior (mean $p_{\text {(long) }}=0.5$ ) before wt or heterozygous animals did (Fig. 7B). From difficulty levels 5 and 4 for the short and long anchor durations, respectively, $p_{(\text {long })}$ was no longer different from 0.5 in the homozygous $\operatorname{tgHD}$, whereas for the same levels of difficulty, wt and heterozygous animals were still showing significant discrimination ( $p_{\text {(long) }}$ different from 0.5 ). These results are in line with the poorer temporal sensitivity of homozygous models observed in the bisection curves.

\section{Neurophysiological assessment of presymptomatic prefrontostriatal functioning}

In this series of experiments, prefrontostriatal synaptic function was assessed through electrophysiological study of the FPs in the DMS evoked by the stimulation of the PL. Initial slope and amplitude of FPs were analyzed. In general, the same pattern of results was observed for both measures, although amplitudes tended to be more variable. We thus choose to report here results obtained with FP slope.

Rats were between 113 and $167 \mathrm{~d}$ old (113-161, 126-165, and 120-167 for wt, heterozygous, and homozygous, respectively). Because the electrophysiological phenotype of transgenic animals may evolve with age, we first tested the homozygous tgHD correlation with age. For this group, there was a tendency toward a negative correlation between age and maximum amplitude or slope of the FPs attained during the I/O protocol. However, this correlation did not reach significance for either measure (FP slope, $n=$ $\left.19, r_{(17)}=0.389, p=0.10\right)$, nor did the percentage of change in the maximum I/O after TBS stimulation correlated with age (FP slope, $n=19, r_{(17)}=0.135$, NS). Therefore, because no clear effect of age was detected, data were regarded as independent of this parameter.

comparisons based on the pseudologistic fit confirmed that homozygous tgHD animals displayed a significantly higher gamma (i.e., a poorer temporal sensitivity) than wt animals $\left(F_{(1,14)}=\right.$ 4.83, $p<0.05$ ) (Fig. 6B). Heterozygous tgHD rats demonstrated an intermediate phenotype with gamma values falling between those of homozygous and wt animals; they were not significantly different from wt rats $\left(F_{(1,13)}=2.1\right.$, NS). There was no significant effect on PSE (all $F$ values $<1$ ) (Fig. 6C).

When the difficulty of the bisection tests was increased by progressively reducing the temporal discriminability among stimulus durations, the bisection curves of the animals flattened
For the I/O curve, all FPs showed a monotonic increase in slope with an increase in stimulation intensity and reached a plateau from $500 \mu \mathrm{A}$ on average. FP slopes for the three groups of rats (wt, homozygous, and heterozygous) differed significantly (group effect, $F_{(2,37)}=3.66, p<0.05$; group $\times$ intensity interaction, $\left.F_{(2,407)}=1.63, p<0.05\right)$. These effects were mainly attributable to the heterozygous tgHD FP slopes, which showed a marked reduction compared with wt (group $\times$ intensity interaction, $F_{(1,19)}=6.38, p=0.02$ ), whereas homozygous animals did not differ from wt controls for FP slope (group $\times$ intensity interaction, $F<1$ ). Thus, 
basal synaptic transmission does not appear to be affected in homozygous transgenic animals.

The paired-pulse function tests plasticity at very short millisecond-range intervals. Paired-pulse facilitation is thought to reflect presynaptic probability of neurotransmitter release (Zucker, 1973; Zucker and Regehr, 2002). Although wt and heterozygous animals showed no significant change in pairedpulse ratios as a function of intervals (interaction of $\mathrm{P} 1 / \mathrm{P} 2 \times$ interval, $F<1$ and $F_{(6,48)}=1.68$, NS, for wt and heterozygous animals, respectively), homozygous tgHD animals showed significant pairedpulse alteration (interaction of $\mathrm{P} 1 / \mathrm{P} 2 \times$ interval, $F_{(6,108)}=3.82, p<0.01$ ) (Fig. $8 C)$. Additional analyses confirmed that, for this latter group only, there was significant paired-pulse facilitation at interstimuli intervals of $20 \mathrm{~ms}\left(F_{(1,18)}=7.96\right.$, $p=0.01)$ and $50 \mathrm{~ms}\left(F_{(1,18)}=8.90, p<\right.$ $0.01)$.

Short-term and long-term plasticity at the prefrontostriatal afferents were analyzed by testing the effect of TBS. As seen on Figure $8 A$, TBS tended to produce a temporary decrease in FP slope that was more pronounced in homozygous rats. Statistical analyses per 10 min blocks confirmed that homozygous rats expressed a significant short-term depression (first 10 min after TBS compared with the $10 \mathrm{~min}$ immediately preceding the TBS, $F_{(1,18)}=$ 6.62, $p<0.02$ ) (Fig. $8 E$ ), which lasted up to 50 min after TBS $\left(F_{(1,18)}=4.78, p<\right.$ $0.05)$. Although a similar tendency was observed in heterozygous rats, no significant depression was found in heterozygous animals $\left(F_{(1,8)}<3.85\right.$, NS $)$ nor in wt $(F<1)$. The long-term effect of TBS was further assessed through changes in paired-pulse and I/O functions. Pairedpulse curves were not modified by the TBS for either group (Fig. 8C) (no interaction of post-TBS/pre-TBS $\times$ interval, $F<1$, $F_{(6,108)}=1.49$ and $F_{(6,48)}=1.12$, NS, for wt, heterozygous, and homozygous transgenic groups, respectively). However, I/O curves were increased after TBS (Fig. $8 \mathrm{~B}$ ). The effect was more pronounced for higher intensities, as evidenced by a pre-TBS/post-TBS $\times$ intensity significant interaction for each group $\left(-/-, F_{(11,121)}=\right.$ $3.48 ;+/-, F_{(11,88)}=1.98 ;+/+, F_{(11,198)}=4.81$; all $p$ values $<$ $0.05)$. Because maximum may not be reached at the same intensity for every animal, we estimated the percentage change at the maximal FP slope value (saturation level) obtained during the I/O curves before and after TBS. This analysis showed that there was a significant potentiation for the homozygous transgenic group $\left(F_{(1,18)}=5.61, p<0.03\right)$ (Fig. $\left.8 D\right)$, although no significant change was observed in wt controls $(F<1)$. The percentage change for the heterozygous group was at an inter-
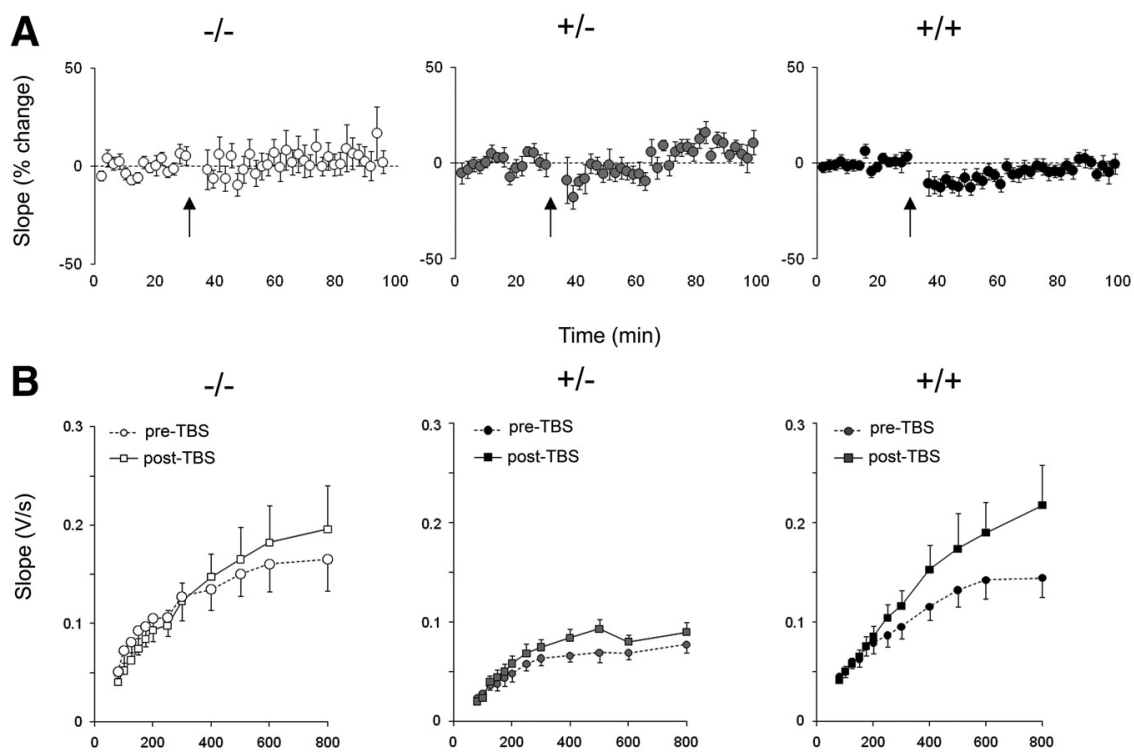

$+/-$
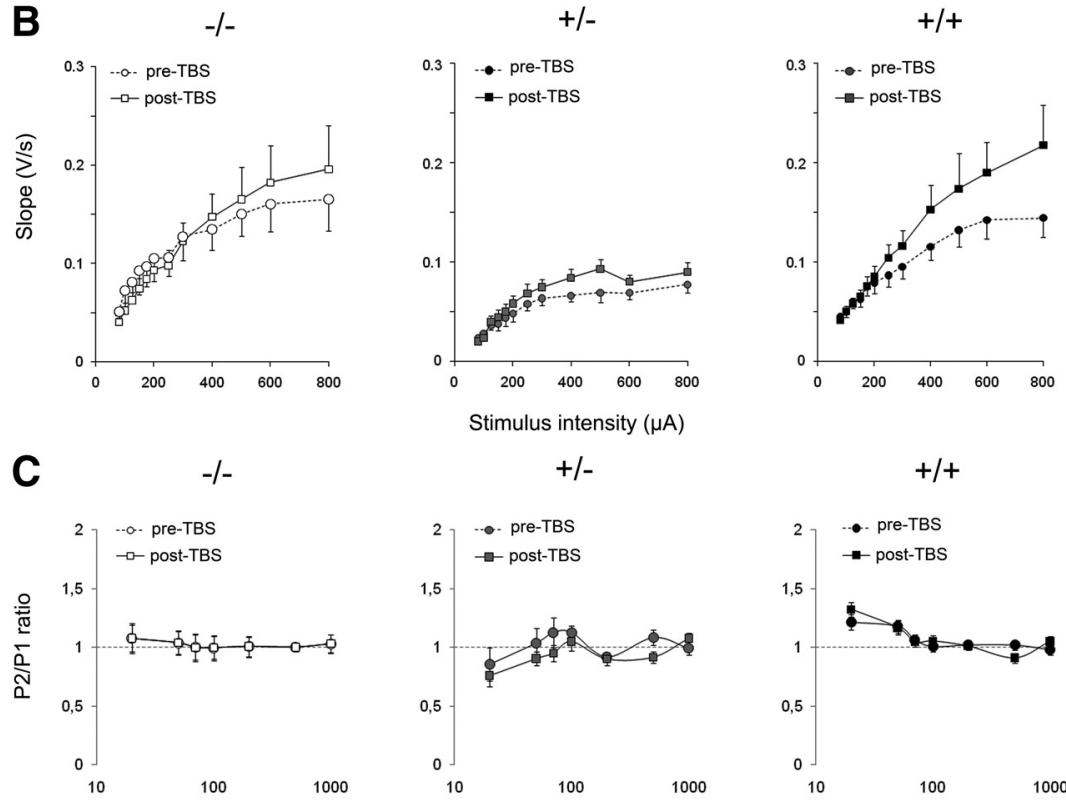

$+/-$
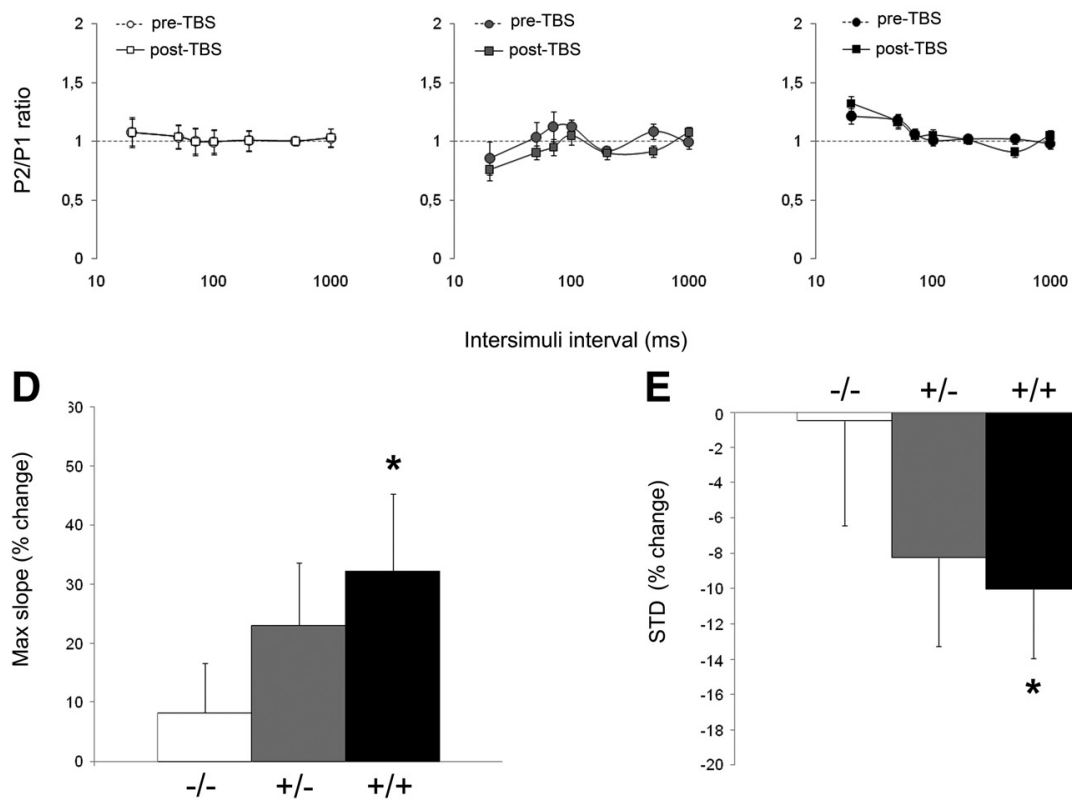

ntersimuli interval (ms)
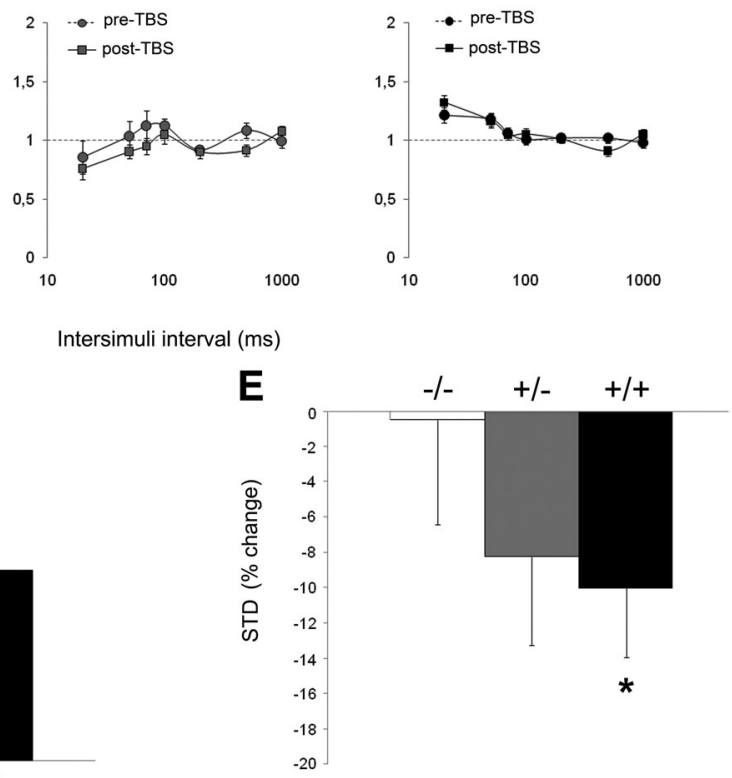

Figure 8. Effect of genotype on prefrontostriatal synaptic transmission in vivo. Comparison of FPs in three groups: wt (-/-, white), homozygous $(+/+$, black), and heterozygous $(+/-$, gray) tgHD rats. $A$, Mean \pm SEM percentage change in FP slope intensity are shown before (pre; dashed lines) and $60-90 \mathrm{~min}$ after (post; solid lines) TBS. C, Paired-pulse ratios for the FP slope as a function of interstimuli intervals (in milliseconds) before (pre; dashed lines) and 60-90 min after (post; solid lines) TBS. D. Mean \pm SEM percentage change induced by TBS in the maximal slope attained during input/output protocols for the three groups of rats. $E$, Short-term plasticity as measured by the mean percentage change during the first 10 min after TBS. ${ }^{*} p<0.05$.

mediate level between wt and homozygous rats, but the potentiation did not reach statistical significance $\left(F_{(1,8)}=2.96, \mathrm{NS}\right)$. Because the potentiation was evidenced mainly at high intensities and the time course of the changes were tested at lower test intensities (mean of 285, 267, and $274 \mu \mathrm{A}$ for wt, heterozygous, and homozygous tgHD rats), whether the potentiation of the saturation level was induced immediately after TBS or whether an initial depression progressively evolved into a potentiation cannot be ascertained with our current data. In any case, the present data show that the homozygous transgenic animals demonstrate an increased plasticity, both short and long term. 


\section{Discussion}

In the search for presymptomatic frontostriatal dysfunction in Huntington disease, we have tested temporal perception in a supra-second range and plasticity at prefrontal afferents to the dorsal striatum in the tgHD rat. Using a bisection task, we show that, as early as 4 months of age, homozygous transgenic animals have a poorer temporal sensitivity than wild-type controls. In vivo analysis of FP in age-matched animals revealed an enhanced plasticity at prefrontostriatal afferents in homozygous transgenic rats.

\section{Symptomatic-related change in temporal behavior}

Previous characterizations of the tgHD rats have shown that the first signs of motor deficits indicators of striatal degeneration emerge at $\sim 6$ months (Nguyen et al. 2006). In line with these neuropathological reports, the present results showing retarded discrimination reversal in transgenic animals at 10 months of age corroborate the striatal damage. This is also in accordance with the bisection effects reported in rats given neurotoxic regimens of methamphetamine thought to produce selective striatal damage (Cheng et al., 2007). Furthermore, motor deficits in these same tgHD rats were also shown to emerge between 7 and 11 months of age (Faure et al., 2011). However, in line with previous reports (Kántor et al., 2006), there was no gross motor disruption because the response latencies were unaltered. At 12 months of age, transgenic rats showed a profound disruption of temporal behavior in the bisection task. Some animals (3 of 12) showed a drastic reduction of temporal discrimination, to the point that their bisection functions were altered and resulted in a " $z$-shaped" function instead of the classical sigmoidal profile. Such a $\mathrm{z}$-shape in the duration-bisection task has been proposed to appear when the distance between anchor durations is reduced, increasing the difficulty of the temporal discrimination (Penney et al., 2008). This suggests that temporal sensitivity was poorer in the aforementioned animals, preventing them from performing appropriately when challenged by a reduced difference between anchor durations (2.64 and $6.06 \mathrm{~s}$ ).

The remaining nine transgenic animals also showed a disruption, reflected by a shift to the right of the bisection function. This cannot be attributable to spatial or position biases, because the duration-lever position combination was counterbalanced between animals within each group. Instead, the shift could reflect a "short bias," a behavior difficult to interpret but that may possibly be related to a differential sensitivity to the reinforcement contingencies (Stubbs, 1968). Alternatively, the shift in PSE may reflect altered storage in temporal reference memory (Meck, 1983). A slower learning of temporal control with delayed peak times has been reported in symptomatic R6/2 mice in a peak interval task (Balci et al., 2009), although the authors did not attribute these deficits to temporal memory. We should note that the task they used had a substantial motor demand and was sensitive to nontemporal factors, such as behavioral inhibition, which may prevent isolation of temporal memory deficits. In our experiment, animals had already been trained for the bisection task at a presymptomatic age and were retrained immediately before bisection testing, allowing adjustments of potential differential clock speed, thus ruling out the clock as opposed to temporal memory. Because no shift in PSE was observed in 4-month-old rats and given the critical role of the dorsal striatum in temporal processing (Coull et al., 2011), the deficit observed in 12-month-old rats is likely attributable to the progressive striatal degeneration.

\section{Altered temporal sensitivity as a presymptomatic marker}

Analysis of the bisection curves in presymptomatic animals showed mild but systematically poorer temporal sensitivity (higher gamma) in homozygous transgenic rats in two independent experiments. The temporal behavior of heterozygous animals was intermediate but not different from controls. The deficit was subtle enough to leave intact the learning rate of the temporal discriminative task, presumably because the initial training was conducted with readily discriminable stimuli ( 2 vs $8 \mathrm{~s}$ ).

Our results demonstrate a presymptomatic timing deficit in a supra-second range, using a cognitive task with low motor demand. In patients, timing capacities have been tested with durations below $1200 \mathrm{~ms}$, with few tasks requiring low motor demand. Among these, a deficit in temporal discrimination has been reported to correlate with estimated age of onset (Paulsen et al., 2004; Beste et al., 2007). When timing was imbedded in motor processes, the deficit was clearly present in pre-HD subjects, even when tested well before the estimated disease onset (Hinton et al., 2007; Paulsen et al., 2008; Rowe et al., 2010). For these subsecond motor timing tasks, increased variability but good timing accuracy was reported. Our results showing poorer temporal sensitivity at a presymptomatic age in a supra-second discrimination task suggest that increased variability in interval timing, independently of modified motor processing, may be a cardinal feature of presymptomatic HD and may participate in the early cognitive impairments.

\section{Altered prefrontostriatal synaptic function}

The electrophysiological results we report here are more complex, because the heterozygous transgenic rats differed from the wt controls with a weaker basal synaptic transmission. However, the general trend observed in the homozygous transgenic rats was an increased plasticity as observed in paired-pulse profiles, shortterm depression, and long-term potentiation.

Electrophysiological studies of HD mouse models have been performed in different brain regions, including the striatum (Zeron et al., 2001; Cepeda et al., 2003, 2007; Kung et al., 2007; Milnerwood and Raymond, 2007) and various cortices (Cummings et al., 2010; Dallérac et al., 2011). Inconsistent results (lower or normal I/O; reduced, normal, or increased paired-pulse facilitation; normal or increased LTD) have been reported, which may be attributable to the difference in animal model but also to the distance from the onset of the degenerative process, because biphasic changes in LTD have been demonstrated in the perirhinal cortex (Cummings et al., 2006). It is not known, however, whether such a biphasic evolution applies to corticostriatal afferents. In agreement with the present results, increased pairedpulse facilitation and increased long-term depression have been observed in slices from YAC mice at a presymptomatic age (Milnerwood and Raymond, 2007). In contrast to our results, a reduced LTP has generally been reported, notably in both the prefrontal cortex and the striatum (Kung et al., 2007, Dallérac et al., 2011). The state of inhibition might be responsible for such discrepancy because GABAergic regulation is weakened in slices, whereas barbiturate anesthesia strongly strengthens inhibition.

The only studies performed in the awake subject showed normal I/O but abolished LTP in the motor cortex in symptomatic patients (Crupi et al., 2008) and altered spiking of medium-spiny neurons of the dorsomedial striatum in symptomatic R6/2 and KI mice (Miller et al., 2008) and 10- to 15-month-old tgHD rats (Miller et al., 2010). The altered firing of striatal neurons in the latter studies were mainly interpreted as reflecting a loss of correlated activity in the cortex. It is noteworthy that changes in field 
potentials evoked by prefrontal stimulation likely represent altered function at the level of the striatum regardless of possible modified cortical processes. The current study is therefore the first report of altered plasticity in the striatum in vivo at the presymptomatic stage. One possibility is that tgHD rats show an enhanced plasticity as a result of increased NMDA receptor sensitivity as shown in the YAC mouse model for HD (Zeron et al., 2002, 2004; Milnerwood and Raymond., 2007; Milnerwood et al., 2010). Additionally, because striatal plasticity is heavily influenced by dopaminergic inputs and dopaminergic dependent loss of plasticity has been reported in cortical and striatal slices (Cummings et al., 2006, Kung et al., 2007, Dallérac et al., 2011), we may speculate that the tgHD altered plasticity is also attributable to changes in dopaminergic signalization at a presymptomatic stage. However, because of the fact that simultaneous depolarization and hyperpolarization are imbedded in the extracellular field potentials, the question remains whether the observed altered plasticity reflects excitatory inputs to striatal neurons or changes in inhibitory transmission within the striatum. An increased reactivity at prefrontal excitatory inputs may potentially favor glutamate excitotoxicity, a hypothesized mechanism for degenerative processes in HD (Olney et al., 1990; Zeron et al., 2002; Estrada-Sánchez et al., 2009). A change in striatal inhibitory control would affect the striatal output.

Interestingly, the enhanced prefrontostriatal plasticity we observe in the current study corroborates the higher degree of activation reported in the cingulate cortex of presymptomatic patients far from disease onset (>12 years) (Paulsen et al., 2004). Such facilitation may reflect early compensatory processes. Alternatively, the enhanced plasticity observed in homozygous tgHD rats may be a consequence of higher glutamine stores (for review, see Antzoulatos and Byrne, 2004), because polyglutamine proteins have been proposed to serve as intracellular storage of glutamine and thereby positively influence learning (Brusilow, 2006). Hence, presymptomatic HD may be associated with enhanced plasticity until degradation of the large polyglutamine tracts by the proteasomes and lysosomes becomes overwhelmed, thus resulting in degenerative changes and impaired plasticity.

\section{Concluding remarks}

The present study provides the first behavioral and electrophysiological evidence of a presymptomatic alteration of prefrontostriatal processing in $\mathrm{HD}$. Whether these modifications are present from birth or have developed earlier will be a critical question to answer if one hopes to use supra-second timing as an early predictive marker of the disease progression. Although it remains to be understood how altered prefrontostriatal function, as evidenced through neurophysiological tests, is responsible for the observed modifications at the behavioral level, our results provide data suggesting that normal plasticity in prefrontostriatal circuits may be necessary for reliable and precise timing behavior.

\section{References}

Allan LG (2002) The location and interpretation of the bisection point. Q J Exp Psychol B 55:43-60.

Antzoulatos EG, Byrne JH (2004) Learning insights transmitted by glutamate. Trends Neurosci 27:555-560.

Balci F, Day M, Rooney A, Brunner D (2009) Disrupted temporal control in the R6/2 mouse model of Huntington's disease. Behav Neurosci 123:1353-1358.

Beste C, Saft C, Andrich J, Müller T, Gold R, Falkenstein M (2007) Time processing in Huntington's disease: a group-control study. PLoS One 2:e1263.

Brusilow WS (2006) Is Huntington a glutamine storage disease? Neuroscientist 12:300-304.
Buhusi CV, Meck WH (2005) What make us tick? Functional and neural mechanisms of interval timing. Nat Rev Neurosci 6:755-765.

Callu D, El Massioui N, Dutrieux G, Brown BL, Doyere V (2009) Cognitive processing impairments in a supra-second temporal discrimination task in rats with cerebellar lesion. Neurobiol Learn Mem 91:250-259.

Cepeda C, Hurst RS, Calvert CR, Hernández-Echeagaray E, Nguyen OK, Jocoy E, Christian LJ, Ariano MA, Levine MS (2003) Transient and progressive electrophysiological alterations in the corticostriatal pathway in a mouse model of Huntington's disease. J Neurosci 23:961-969.

Cepeda C, Wu N, André VM, Cummings DM, Levine MS (2007) The corticostriatal pathway in Huntington's disease. Prog Neurobiol 81:253-271.

Charpier S, Deniau JM (1997) In vivo activity-dependent plasticity at cortico-striatal connections: evidence for physiological long-term potentiation. Proc Natl Acad Sci U S A 94:7036-7040.

Charpier S, Mahon S, Deniau JM (1999) In vivo induction of striatal longterm potentiation by low-frequency stimulation of the cerebral cortex. Neuroscience 91:1209-1222.

Cheng RK, MacDonald CJ, Meck WH (2006) Differential effects of cocaine and ketamine on time estimation: Implications for neurobiological models of interval timing. Pharmacol Biochem Behav 85:114-122.

Cheng RK, Etchegaray M, Meck WH (2007) Impairments in timing, temporal memory, and reversal learning linked to neurotoxic regimens of methamphetamine intoxication. Brain Res 1186:255-266.

Church RM, Deluty MZ (1977) Bisection of temporal intervals. J Exp Psychol Anim Behav Process 3:216-228.

Coull JT, Cheng RK, Meck WH (2011) Neuroanatomical and neurochemical substrates of timing. Neuropsychopharmacology 36:3-25.

Crupi D, Ghilardi MF, Mosiello C, Di Rocco A, Quartarone A, Battaglia F (2008) Cortical and brainstem LTP-like plasticity in Huntington's disease. Brain Res Bull 75:107-114.

Cummings DM, Milnerwood AJ, Dallérac GM, Waights V, Brown JY, Vatsavayai SC, Hirst MC, Murphy KP (2006) Aberrant cortical synaptic plasticity and dopaminergic dysfunction in a mouse model of Huntington's disease. Hum Mol Genet 15:2856-2868.

Cummings DM, Cepeda C, Levine MS (2010) Alterations in striatal synaptic transmission are consistent across genetic mouse models of Huntington's disease. ASN Neuro 2:e00036.

Dallérac GM, Vatsavayai SC, Cummings DM, Milnerwood AJ, Peddie CJ, Evans KA, Walters SW, Rezaie P, Hirst MC, Murphy KP (2011) Impaired long-term potentiation in the prefrontal cortex of Huntington's disease mouse models: rescue by D1 dopamine receptor activation. Neurodegener Dis 8:230-239.

Estrada-Sánchez AM, Montiel T, Segovia J, Massieu L (2009) Glutamate toxicity in the striatum of the R6/2 Huntington's disease transgenic mice is age-dependent and correlates with decreased levels of glutamate transporters. Neurobiol Dis 34:78-86.

Faure A, Höhn S, Von Hörsten S, Delatour B, Raber K, Le Blanc P, Desvignes N, Doyère V, El Massioui N (2011) Altered emotional and motivational processing in the transgenic rat model for Huntington's disease. Neurobiol Learn Mem 95:92-101.

Fitch RH, Threlkeld SW, McClure MM, Peiffer AM (2008) Use of a modified prepulse inhibition paradigm to assess complex auditory discrimination in rodents. Brain Res Bull 76:1-7.

Foroud T, Siemers E, Kleindorfer D, Bill DJ, Hodes ME, Norton JA, Conneally PM, Christian JC (1995) Cognitive scores in carriers of Huntington's disease gene compared to noncarriers. Ann Neurol 37:657-664.

Gabbott PL, Warner TA, Jays PR, Salway P, Busby SJ (2005) Prefrontal cortex in the rat: projections to subcortical autonomic, motor, and limbic centers. J Comp Neurol 492:145-177.

Gerfen CR (1984) The neostriatal mosaic: compartmentalization of corticostriatal input and striatonigral output systems. Nature 311:461-464.

Grondin S (2001) Discriminating time intervals presented in sequences marked by visuals signals. Percept Psychophys 63:1214-1228.

Hinton SC, Paulsen JS, Hoffmann RG, Reynolds NC, Zimbelman JL, Rao SM (2007) Motor timing variability increases in preclinical Huntington's disease patients as estimated onset of motor symptoms approaches. J Int Neuropsychol Soc 13:539-543.

Kántor O, Temel Y, Holzmann C, Raber K, Nguyen HP, Cao C, Türkoglu HO, Rutten BP, Visser-Vandewalle V, Steinbusch HW, Blokland A, Korr H, Riess O, von Hörsten S, Schmitz C (2006) Selective striatal neuron loss and alterations in behavior correlate with impaired striatal function in Huntington's disease transgenic rats. Neurobiol Dis 22:538-547. 
Karl T, Pabst R, von Hörsten S (2003) Behavioral phenotyping of mice in pharmacological and toxicological research. Exp Toxicol Pathol 55:69-83.

Killeen PR, Fetterman JG, Bizo LA (1997) Time's causes. In: Time and behavior: psychological and neurological analyses (Bradshaw CM, Szabadi E, eds), pp 79-131. Amsterdam: Elsevier Science.

Kung VW, Hassam R, Morton AJ, Jones S (2007) Dopamine-dependent long term potentiation in the dorsal striatum is reduced in the R6/2 mouse model of Huntington's disease. Neuroscience 146:1571-1580.

Lawrence AD, Sahakian BJ, Hodges JR, Rosser AE, Lange KW, Robbins TW (1996) Executive and mnemonic functions in early Huntington's disease. Brain 119:1633-1645.

Lawrence AD, Hodges JR, Rosser AE, Kershaw A, ffrench-Constant C, Rubinsztein DC, Robbins TW, Sahakian BJ (1998) Evidence for specific cognitive deficits in preclinical Huntington's disease. Brain 121:13291341.

Maricq AV, Church RM (1983) The differential effects of haloperidol and methamphetamine on time estimation in the rat. Psychopharmacology 79:10-15.

Maricq AV, Roberts S, Church RM (1981) Methamphetamine and time estimation. J Exp Psychol Anim Behav Process 7:18-30.

Matell MS, Bateson M, Meck WH (2006) Single-trials analyses demonstrate that increases in clock speed contribute to the methamphetamineinduced horizontal shifts in peak-interval timing functions. Psychopharmacology 188:201-212.

Meck WH (1983) Selective adjustment of the speed of internal clock and memory processes. J Exp Psychol Anim Behav Process 9:171-201.

MeckWH (2006) Neuroanatomical localization of an internal clock: a functional link between mesolimbic, nigrostriatal, and mesocortical dopaminergic systems. Brain Res 1109:93-107.

Miller BR, Walker AG, Fowler SC, von Hörsten S, Riess O, Johnson MA, Rebec GV (2010) Dysregulation of coordinated neuronal firing patterns in striatum of freely behaving transgenic rats that model Huntington's disease. Neurobiol Dis 37:106-113.

Miller BR, Walker AG, Shah AS, Barton SJ, Rebec GV (2008) Dysregulated information processing by medium spiny neurons in striatum of freely behaving mouse models of Huntington's disease. J Neurophysiol 100:2205-2216.

Milnerwood AJ, Raymond LA (2007) Corticostriatal synaptic function in mouse models of Huntington's disease: early effects of huntingtin repeats length and protein load. J Physiol 585:817-831.

Milnerwood AJ, Cummings DM, Dallérac GM, Brown JY, Vatsavayai SC, Hirst MC, Rezaie P, Murphy KP (2006) Early development of aberrant synaptic plasticity in a mouse model of Huntington's disease. Hum Mol Genet 15:1690-1703.

Milnerwood AJ, Gladding CM, Pouladi MA, Kaufman AM, Hines RM, Boyd JD, Ko RW, Vasuta OC, Graham RK, Hayden MR, Murphy TH, Raymond LA (2010) Early increases in extrasynaptic NMDA receptor signaling and expression contributes to phenotype onset in Huntington's disease mice. Neuron 65:178-190.

Nguyen HP, Kobbe P, Rahne H, Wörpel T, Jäger B, Stephan M, Pabst R, Holzmann C, Riess O, Korr H, Kántor O, Petrasch-Parwez E, Wetzel R, Osmand A, von Hörsten S (2006) Behavioral abnormalities precede neuropathological markers in rats transgenic for Huntington's disease. Hum Mol Genet 15:3177-3194.

Olney JW, Zorumski CF, Stewart GR, Price MT, Wang GJ, Labruyere J (1990) Exitotoxicity of L-dopa and 6-OH-dopa: implications for Parkinson's and Huntington's diseases. Exp Neurol 108:269-272.

Paulsen JS, Zimbelman JL, Hinton SC, Langbehn DR, Leveroni CL, Benjamin ML, Reynolds NC, Rao SM (2004) fMRI biomarker of early neuronal dysfunction in presymptomatic Huntington's disease. Am J Neuroradiol 25:1715-1721.

Paulsen JS, Langbehn DR, Stout JC, Aylward E, Ross CA, Nance M, Guttman M, Johnson S, MacDonald M, Beglinger LJ, Duff K, Kayson E, Biglan K, Shoulson I, Oakes D, Hayden M; Predict-HD Investigators and Coordinators of the Huntington Study Group (2008) Detection of Huntington's disease decades before diagnosis: the predict-HD study. J Neurol Neurosurg Psychiatry 79:874-880.

Paxinos G, Watson C (1986) The rat brain in stereotaxic coordinates. San Diego: Academic.

Penney TB, Gibbon J, Meck WH (2008) Categorical scaling of duration bisection in pigeons (Colulmbia livia), mice (Mus musculus), and humans (Homo sapiens). Psychol Sci 19:1103-1109.

Rammsayer TH (1999) Neuropharmacological evidence for different timing mechanisms in humans. Q J Exp Psychol B 52:273-286.

Rouanet H, Bernard J-M, Le Roux B (1990) Analyse inductive des données. In: Statistique en sciences humaines. Paris: Dunod.

Rowe KC, Paulsen JS, Langbehn DR, Duff K, Beglinger LJ, Wang C, O’Rourke JJ, Stout JC, Moser DJ (2010) Self-paced timing detects and tracks change in prodromal Huntington disease. Neuropsychology 24:435-442.

Stubbs A (1968) The discrimination of stimulus duration by pigeons. J Exp Anal Behav 11:223-238.

Urbach YK, Bode FJ, Nguyen HP, Riess O, von Hörsten S (2010) Neurobehavioral tests in rat models of degenerative brain diseases. Methods Mol Biol 597:333-356.

von Hörsten S, Schmitt I, Nguyen HP, Holzmann C, Schmidt T, Walther T, Bader M, Pabst R, Kobbe P, Krotova J, Stiller D, Kask A, Vaarmann A, Rathke-Hartlieb S, Schulz JB, Grasshoff U, Bauer I, Vieira-Saecker AM, Paul M, Jones L, Lindenberg KS, Landwehrmeyer B, Bauer A, Li XJ, Riess O (2003) Transgenic rat model for Huntington's disease. Hum Mol Genet 12:617-624.

Vonsattel JP, Myers RH, Stevens TJ, Ferrante RJ, Bird ED, Richardson EP Jr (1985) Neuropathological classification of Huntington's disease. J Neuropathol Exp Neurol 44:559-577.

Walker AG, Miller BR, Fritsch JN, Barton SJ, Rebec GV (2008) Altered information processing in the prefrontal cortex of Huntington's disease mouse models. J Neurosci 28:8973-8982.

Wilson RS, Como PG, Garron DC, Klawans HL, Barr A, Klawans D (1987) Memory failure in Huntington's disease. J Clin Exp Neuropsychol 9:147-154.

Woodruff-Pak DS, Papka M (1996) Huntington's disease and eyeblink classical conditioning: normal learning but abnormal timing. J Int Neuropsychol Soc 2:323-334.

Zeron MM, Chen N, Moshaver A, Lee AT, Wellington CL, Hayden MR, Raymond LA (2001) Mutant huntingtin enhances excitotoxic cell death. Mol Cell Neurosci 17:41-53.

Zeron MM, Hansson O, Chen N, Wellington CL, Leavitt BR, Brundin P, Hayden MR, Raymond LA (2002) Increased sensitivity to $N$-methyl-Daspartate receptor-mediated excitotoxicity in a mouse model of Huntington's disease. Neuron 33:849-860.

Zeron MM, Fernandes HB, Krebs C, Shehadeh J, Wellington CL, Leavitt BR, Baimbridge KG, Hayden MR, Raymond LA (2004) Potentiation of NMDA receptor-mediated excitotoxicity linked with intrinsic apoptotic pathway in YAC transgenic mouse model of Huntington's disease. Mol Cell Neurosci 25:469-479.

Zucker RS (1973) Changes in the statistics of transmitter release during facilitation. J Physiol 229:787-810.

Zucker RS, Regehr WG (2002) Short-term plasticity. Annu Rev Physiol 64: 355-405. 